ARTICLE

Received 21 Oct 2016 | Accepted 18 Jan 2017 | Published 6 Mar 2017

DOI: $10.1038 /$ ncomms14642

OPEN

\title{
Regulation of phagocyte triglyceride by a STAT-ATG2 pathway controls mycobacterial infection
}

Claire B. Péan ${ }^{1}$, Mark Schiebler ${ }^{2}$, Sharon W.S. Tan ${ }^{3}$, Jessica A. Sharrock ${ }^{1}$, Katrin Kierdorf ${ }^{1}$, Karen P. Brown ${ }^{2,4}$, M. Charlotte Maserumule ${ }^{2}$, Shinelle Menezes ${ }^{3}$, Martina Pilátová $^{5}$, Kévin Bronda ${ }^{3}$, Pierre Guermonprez ${ }^{3}$, Brian M. Stramer ${ }^{5}$, R. Andres Floto ${ }^{2,4} \&$ Marc S. Dionne ${ }^{1}$

Mycobacterium tuberculosis remains a global threat to human health, yet the molecular mechanisms regulating immunity remain poorly understood. Cytokines can promote or inhibit mycobacterial survival inside macrophages and the underlying mechanisms represent potential targets for host-directed therapies. Here we show that cytokine-STAT signalling promotes mycobacterial survival within macrophages by deregulating lipid droplets via ATG2 repression. In Drosophila infected with Mycobacterium marinum, mycobacterium-induced STAT activity triggered by unpaired-family cytokines reduces Atg2 expression, permitting deregulation of lipid droplets. Increased Atg2 expression or reduced macrophage triglyceride biosynthesis, normalizes lipid deposition in infected phagocytes and reduces numbers of viable intracellular mycobacteria. In human macrophages, addition of IL-6 promotes mycobacterial survival and BCG-induced lipid accumulation by a similar, but probably not identical, mechanism. Our results reveal Atg2 regulation as a mechanism by which cytokines can control lipid droplet homeostasis and consequently resistance to mycobacterial infection in Drosophila.

\footnotetext{
${ }^{1}$ MRC Centre for Molecular Bacteriology and Infection, and Department of Life Sciences, Imperial College London, Ground Floor, Flowers Building, South Kensington Campus, London SW7 2AZ, UK. ${ }^{2}$ Molecular Immunity Unit, MRC Laboratory of Molecular Biology, University of Cambridge Department of Medicine, Cambridge CB2 OQH, UK. ${ }^{3}$ Centre for Molecular and Cellular Biology of Inflammation, School of Medicine, King's College London, London SE1 1UL, UK. ${ }^{4}$ Cambridge Centre for Lung Infection, Papworth Hospital, Cambridge CB23 3RE, UK. ${ }^{5}$ Randall Division of Cell and Molecular Biophysics, King's College London, London SE1 1UL, UK. Correspondence and requests for materials should be addressed to M.S.D. (email: m.dionne@imperial.ac.uk).
} 
$\mathrm{V}$ irulent mycobacteria are critically dependent on their ability to survive within macrophages to cause disease. The tendency of macrophages to support or kill mycobacteria is directly regulated by distinct cytokine-JAKSTAT pathways: for example, the Th1 cytokine interferonrimproves the ability of the macrophage to kill mycobacteria, whereas interleukin (IL)-10 and the Th2-derived cytokines IL-4 and IL-13 can inhibit killing of intracellular mycobacteria ${ }^{1-3}$. Our knowledge of the in vivo functions of these signals in mycobacterial infection, and of their transcriptional targets and the associated physiologies, remains incomplete, especially for those signals that inhibit killing of intracellular bacteria ${ }^{4,5}$. The genetic and cell-biological mechanisms that underlie these responses are potentially fertile ground for host-targeted therapies for tuberculosis 6,7 .

The fruitfly Drosophila melanogaster is a well-established model for the study of innate immunity ${ }^{8}$. Many aspects of the innate immune response to microbes are conserved between flies and vertebrates, including the central role of nuclear factor- $\kappa \mathrm{B}$ family transcription factors and Toll-like receptors, antiinflammatory effects of transforming growth factor- $\beta$ family signals and the presence of bactericidal phagocytes homologous to vertebrate macrophages ${ }^{9-11}$. We have developed a system in which Drosophila are infected with Mycobacterium marinum, which allows us to identify host mechanisms involved in mycobacterial infection ${ }^{12,13}$. As in mammals, cytokine production by phagocytes is a critical component of the cellular response to infection. Flies have three known genes encoding ILlike signals, upd1, upd2 and upd3 (ref. 14). These proteins have structural and functional similarity to the four-helix-bundle cytokines of mammals ${ }^{15}$. They bind a GP130-like receptor, encoded by domeless, to activate a conserved JAK-STAT signalling cassette $\mathrm{e}^{16}$. Upon bacterial infection in adult flies, upd3 is expressed by phagocytes and activates the JAK-STAT pathway in the fat body, where it is required for infectioninduced expression of the stress peptide Tot ${ }^{14}$. This signalling pathway is critically required for antiviral defense ${ }^{17}$, it regulates larval haematopoiesis ${ }^{18}$ and it is important in maintaining gut integrity in response to bacterial infection and other stresses ${ }^{19-23}$. Other roles in immune defense against bacteria are unknown.

Here we show that unpaired-family cytokines are critical regulators of defense against intracellular virulent mycobacteria. We show that M. marinum infection drives production of upd 3 in phagocytes, and that reception of this signal by phagocytes is detrimental to the host. JAK-STAT blockade increased resistance to infection: it prolonged survival of the host, reduced mycobacterial numbers and delayed immune cell death, with similar effects observed in vitro. This effect was associated with overexpression of the autophagy-related gene Atg2 and not other autophagy genes, in vivo and in vitro. M. marinum infection of cultured phagocytes increased intracellular neutral lipids and drove accumulation of unusually large lipid droplets (LDs). Atg2 overexpression or JAK-STAT inhibition reduced intracellular mycobacterial number and partially normalized phagocyte lipid droplet size without significantly changing bulk autophagy. Direct inhibition of triglyceride synthesis prevented the infectioninduced alteration in LDs, reduced mycobacteria-LD association and reduced intracellular viable mycobacteria. We then tested these findings in human cells. Similar to Drosophila, IL-6 signalling in macrophages increased intracellular Bacillus Calmette-Guérin (BCG) and Mycobacterium tuberculosis numbers in a dose-dependent manner; ATG2A expression was also reduced, although only to a very small extent. Importantly, the ability of IL- 6 to increase the number of viable intracellular mycobacteria was abolished by inhibition of macrophage triglyceride synthesis. Together, these data indicate that loss of a STAT-activating cytokine pathway can reduce survival of intracellular mycobacteria via effects on cellular lipid deposition. In Drosophila, one responsible effector may be ATG2.

\section{Results}

unpaired signalling decreases resistance to $M$. marinum. The IL-6-like cytokine upd3 can be induced in haemocytes by infection with non-pathogenic bacteria; however, the ability of bacterial pathogens to induce its expression has not been previously explored ${ }^{14}$. This was particularly in question with M. marinum, which is not a strong agonist of known patternrecognition pathways in Drosophila ${ }^{12}$. We thus examined the in vivo expression of $u p d 3$ during the initial phase of $M$. marinum infection using an upd3 green fluorescent protein (GFP) reporter and a haemocyte-specific dsRed nuclear marker ${ }^{10,14}$. We observed strong GFP induction in adult Drosophila haemocytes after mycobacterial infection (Fig. 1a), indicating that mycobacterial infection was indeed sufficient to drive upd3 production from macrophages in vivo. This effect was also visible by quantitative reverse transcriptase-PCR (qRT-PCR) in samples from whole flies (Fig. 1b).

To test the role of $u p d 3$ in mycobacterial infection in Drosophila, we infected flies carrying the $o s^{s}$ mutation with M. marinum. os $s^{s}$ affects expression of the cytokines upd 1 and $u p d 3$, and impairs induction of upd3 by infection with nonpathogenic bacteria ${ }^{14,24}$. We observed that $o s^{s}$ mutants did not activate $u p d 3$ expression to the same degree as wild-type flies after $M$ marinum infection, whereas upd1 expression was not induced by $M$. marinum infection in wild-type flies or $o s^{s}$ mutants (Fig. 1b). Despite identical starting inocula, $o s^{s}$ mutants carried a much lower mycobacterial burden at late stages of infection and survived longer than wild-type controls (Fig. 1c,d).

Although haemocytes were the only visible site of upd3 induction in M. marinum-infected $u p d 3>$ GFP flies, it remained possible that some other tissue was contributing to the relevant UPD3 pool. Moreover, although we saw no significant induction of upd1 by infection, changes in UPD1 might still contribute to the $o s^{s}$ phenotype. To verify that haemocyte-derived UPD3 was responsible for the mycobacterial resistance phenotype of $o s^{s}$ mutants, we knocked down upd3 with the haemocyte-specific crqGal4 driver and examined mycobacterial pathogenesis ${ }^{25}$. Haemocyte upd3 knockdown gave strong lifespan extension after infection and increased mycobacterial number (Fig. 1e,f). Together, these experiments indicate that haemocytes produce upd3 upon mycobacterial infection and haemocyte-derived upd3 shortens the infected lifespan of the host and impairs resistance against M. marinum.

Haemocyte STAT activity decreases resistance to mycobacteria. To identify relevant UPD3 target tissues, we used RNA interference (RNAi) knockdown of Stat92E (the sole Drosophila STAT). Prior work has suggested that immune-induced STAT signalling is important in regulating humoral immune responses via effects on the Drosophila fat body ${ }^{26}$. However, Stat92E knockdown in the fat body had no effect on survival after M. marinum infection (Supplementary Fig. 1a). The primary direct immune effect of the fat body is the immune-inducible expression of antimicrobial peptides; we found that $o s^{s}$ mutant flies exhibited no consistent differences in antimicrobial peptide expression before haemocyte death (Supplementary Fig. 1b-e). We thus concluded that the effect of upd3 on mycobacterial infection was independent of the fat body.

We next tested the effect of cytokine signalling on haemocytes themselves. As the JAK-STAT pathway regulates larval haematopoiesis, we combined our haemocyte drivers with $G a l 80^{\text {ts }}$, 
making their effect temperature sensitive, to enable adult-specific Stat92E knockdown ${ }^{18,27,28}$. Adult-specific Stat $92 E$ knockdown or expression of a dominant-negative form of the upd3 receptor dome in haemocytes significantly improved resistance to infection (Fig. 2a,b and Supplementary Fig. 2). These results together indicate that haemocyte-derived $u p d 3$ activates STAT signalling in haemocytes, decreasing resistance to mycobacterial infection and impairing host survival.

Cell death via necrosis or apoptosis is commonly observed in vertebrate macrophages infected with $M$. tuberculosis or M. marinum ${ }^{29-31}$. We observed marked cytotoxicity due to M. marinum infection in Drosophila S2R ${ }^{+}$cells, an embryonicderived macrophage cell line, and progressive loss of haemocytes during the last few days of $M$. marinum infection in vivo
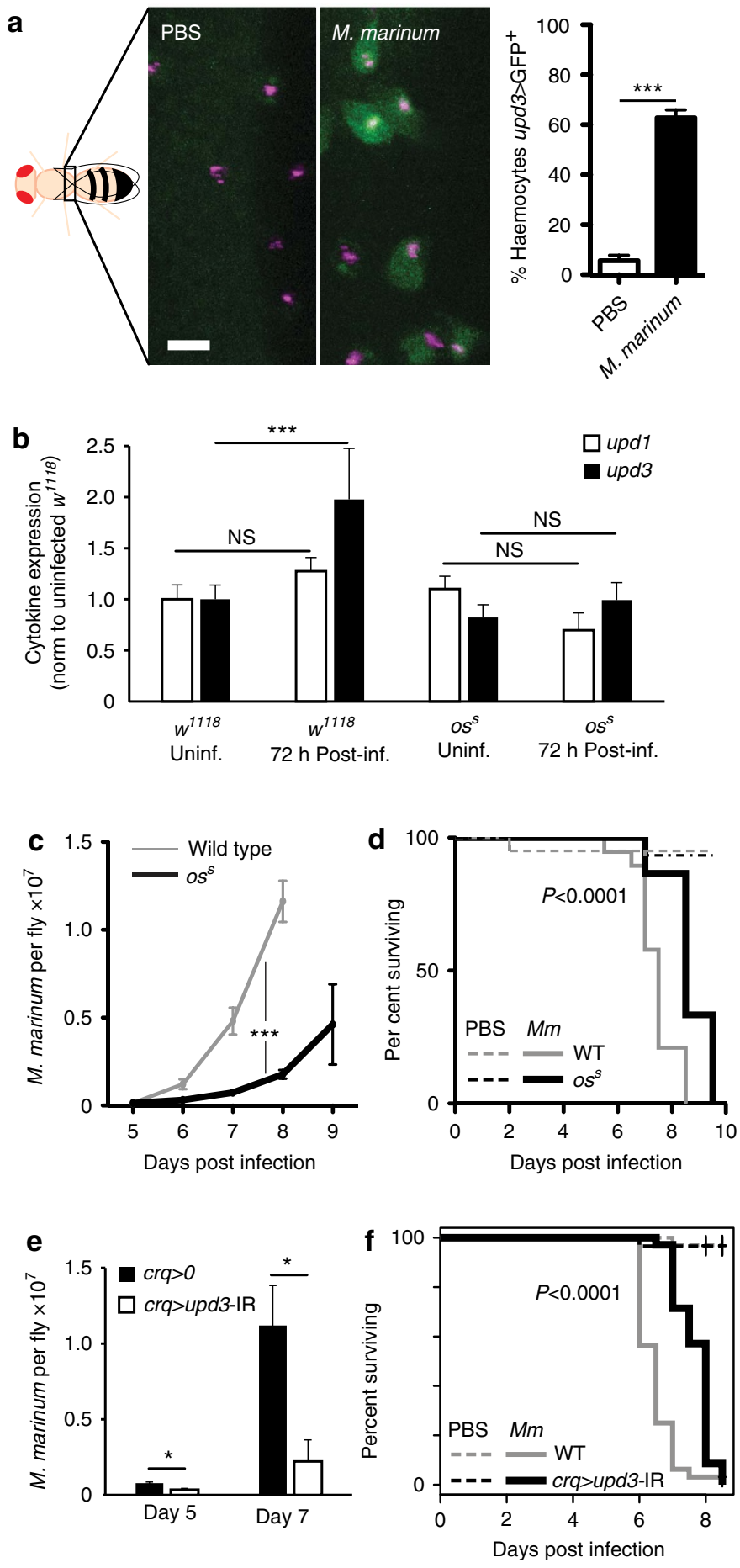

(Supplementary Fig. 3a-d). Just before dying, flies harboured no visible haemocytes and dramatically reduced expression of the haemocyte marker $\mathrm{Hml}$ was detected by qRT-PCR on the whole fly (Supplementary Fig. 3c-e). The os mutation improved haemocyte survival in infected flies (Supplementary Fig. 3b-d). Importantly, this was not a result of differential microbial internalization, as the fraction of haemocytes containing $M$. marinum was not different between control and $u p d 3-I R$ flies $16 \mathrm{~h}$ after infection (Supplementary Fig. 4a,b). The difference in haemocyte survival, such as the difference in haemocyte number, was also seen in flies with adult-specific haemocyte Stat92E knockdown (Supplementary Fig. 4c-e). We thus conclude that upd3-STAT signalling alters the interaction between mycobacteria and phagocytes so as to impair the survival of infected cells.

JAK-STAT activation in haemocytes downregulates Atg2. We wished to identify target genes and cell-biological processes behind the reduced mycobacterial numbers seen as a result of impaired cytokine signalling. Activation of JAK-STAT signalling in Kc167 cells reduces expression of several autophagy-related genes ${ }^{32}$. As phagocyte autophagy can impair intracellular survival of M. tuberculosis ${ }^{3}$, we tested this transcriptional effect in vivo, analysing the expression levels of 14 autophagy-related genes in flies with haemocyte-specific Stat92E knockdown. Surprisingly, Atg2 was the only autophagy gene with strongly enhanced expression following mycobacterial infection in Stat92E knockdown flies (Fig. 2c). Further analysis revealed that Atg2 overexpression was seen in haemocyte-specific Stat $92 E$ and $u p d 3$ knockdown flies independent of infection (Supplementary Fig. 5a,b). Conversely, in vitro, upd3 overexpression induced the known JAK-STAT pathway target TotA and reduced Atg2 expression (Fig. 2d and Supplementary Fig. 5c,d). The specific effect on Atg2 expression was particularly interesting, as in HeLa cells Atg2 is separably important for autophagy and neutral lipid

Figure 1 | The unpaired cytokines reduce resistance to $\boldsymbol{M}$. marinum infection. (a) upd3 expression in haemocytes after M. marinum infection. Left, intravital imaging of eGFP expressed under the control of upd3-Gal4 in flies injected with PBS or M. marinum (5,000 CFU). Haemocytes are labelled with nuclear dsRed directly driven by the $\mathrm{Hml} \Delta$ promoter $^{10}$. This image was taken $48 \mathrm{~h}$ after injection. Scale bar, $10 \mu \mathrm{m}$. Right, quantification of the fraction of GFP-positive cells from seven flies based on images similar to the one shown. Error bars show s.d. Values are statistically different $\left({ }^{\star \star \star} P<0.001\right)$ by Mann-Whitney test. (b) mRNA expression of upd 1 and upd 3 in wild-type $\left(w^{1118}\right)$ and $s^{5}$ flies, uninfected or $72 \mathrm{~h}$ after infection with M. marinum. Assayed by qRT-PCR and normalized to Rp/1 (as a loading control), and then to uninfected wild-type flies for each gene. $n=4$ for each condition. Data were log-transformed before statistical comparison by Student's $t$-test $\left({ }^{\star \star \star} P<0.001\right)$. (c) M. marinum burden in wild-type and $0 s^{s}$ mutant flies, assayed by qRT-PCR. Flies were injected with $500 \mathrm{CFU} M$. marinum and kept at $25^{\circ} \mathrm{C}$. P-value for differences in bacterial numbers by two-way analysis of variance (ANOVA), $n=6$ per time point except for day 9 where $n=3$ for $0 S^{5}$ flies. (d) Survival of wild-type and os ${ }^{5}$-mutant flies, injected with PBS or infected with 500 CFUs M. marinum, as indicated. Flies were kept at $25^{\circ} \mathrm{C}$. P-value for difference between infected survival curves by log-rank test. (e) M. marinum burden in control ( $w^{1118 ; ;}$ crq-Gal4/+) and haemocyte-specific upd3-knockdown ( $w^{1118}$; UAS-upd3-IR/ + ; crq-Gal4/ + ) flies, assayed by qRT-PCR. Flies were injected with $500 \mathrm{CFU}$ M. marinum and kept at $25^{\circ} \mathrm{C}$. Here and elsewhere, 'crq > upd3-IR' is used as an abbreviation for 'crq-Gal4, UAS-upd3-IR'. $P$-value for differences in bacterial numbers by one-way ANOVA, $n=3$ per time point $\left({ }^{\star} P<0.05\right)$. (f) Survival of control ( ${ }^{1118} ; "$ crq-Gal4/ +$)$ and haemocyte-specific upd3-knockdown ( ( $^{1118}$; UAS-upd3-IR/ + ; crq-Gal4/ +) flies, injected with PBS or $500 \mathrm{CFU} M$. marinum. Flies were kept at $25^{\circ} \mathrm{C}$. $P$-value for difference between infected survival curves by log-rank test. 
a

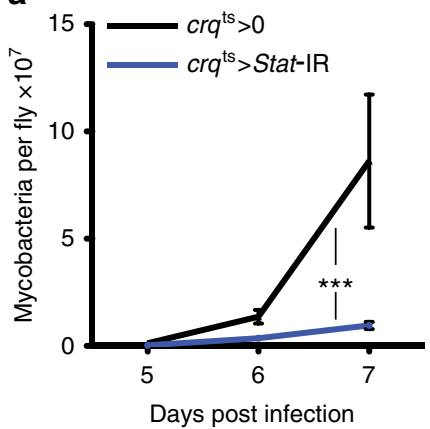

C

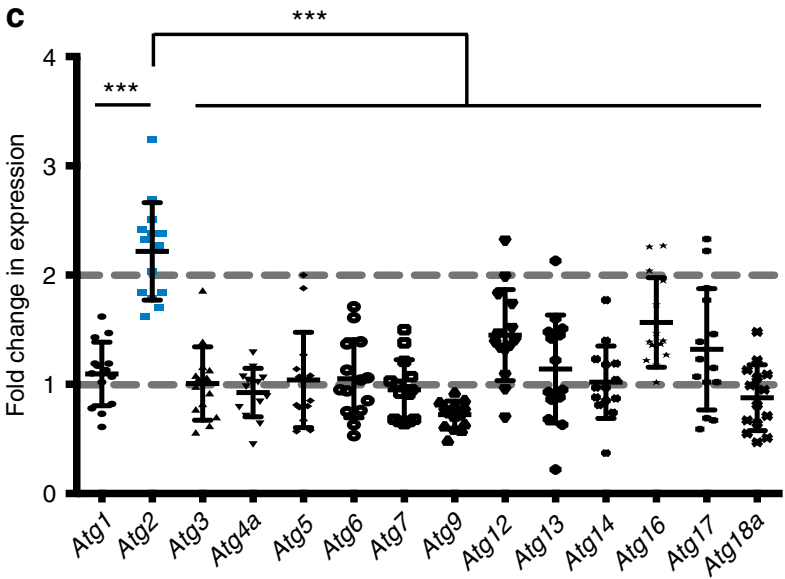

b

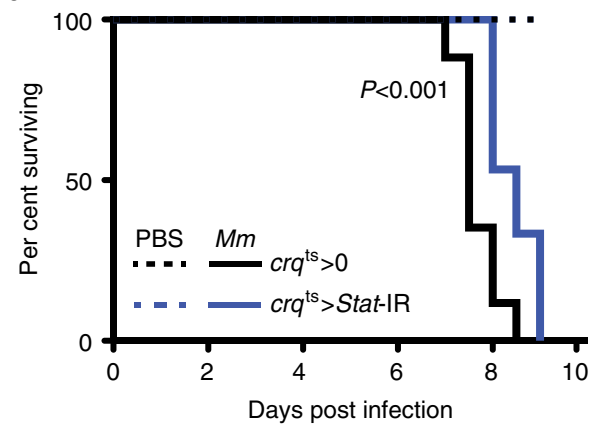

d

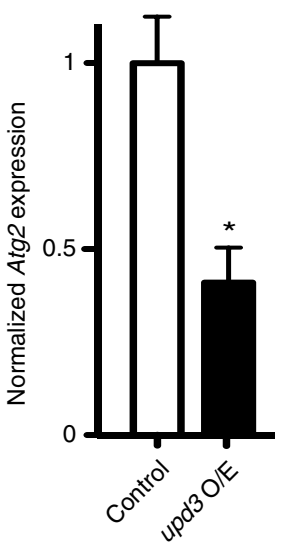

e

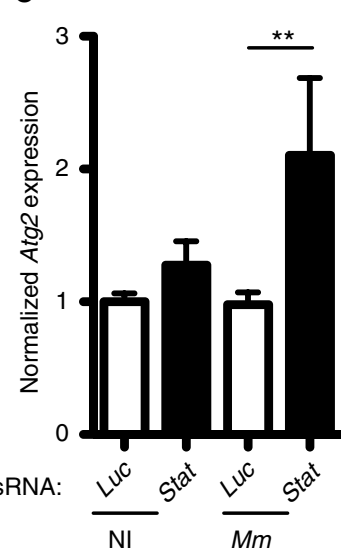

f

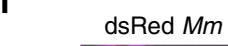

Mm+brightfield
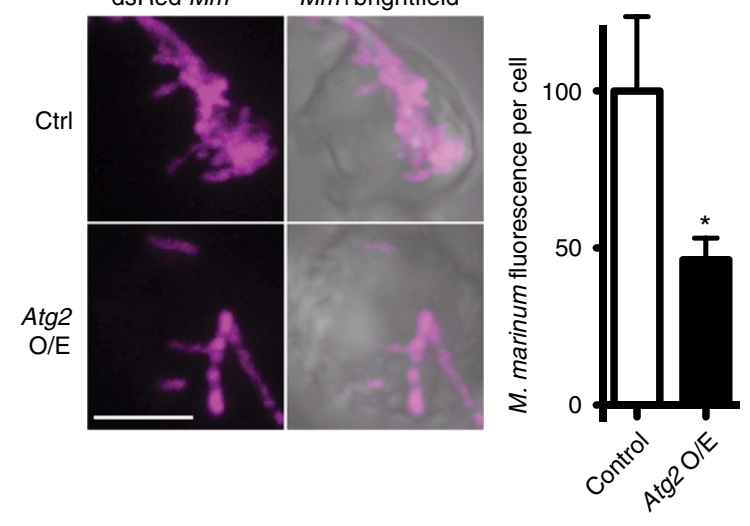

g

Figure 2 | STAT activation in haemocytes impairs Atg2 expression to reduce mycobactericidal activity. (a) M. marinum burden in control ( $w^{1118 ;}$ tub-Gal80ts / + ; crq-Gal4/ +) and inducible haemocyte-specific Stat92E-knockdown ( ${ }^{1718}$, UAS-Stat92E-IR/ + ; UAS-Stat92E-IR/tub-Gal80 $0^{\text {ts; }}$ crq-Gal4/ + ) flies, assayed by qRT-PCR. Flies were initially infected with $500 \mathrm{CFU}$ M. marinum and cultured at $29^{\circ} \mathrm{C}$. $P$-value for difference in bacterial numbers by two-way analysis of variance (ANOVA), $n=5$ per timepoint. (b) Survival of control ( $w^{1118}$; tub-Gal80 ${ }^{\text {ts }} /+$; crq-Gal4/ +) and haemocyte-specific Stat92Eknockdown ( $w^{1118}$, UAS-Stat92E -IR/ +; UAS-Stat92E -IR/tub-Gal80 ts; crq-Gal4/ +) flies, injected with PBS or infected with 500 CFU M. marinum, as indicated. Infectious dose and temperature as in a. $P$-value for difference between infected survival curves by log-rank test. (c) Fold change in expression of several autophagy genes in Stat92E-IR ( $w^{1118}$, UAS-Stat92E-IR; UAS-Stat92E-IR/tub-Gal80ts; crq-Gal4/ +) flies compared with controls ( $w^{1118}$; tub-Gal80 ${ }^{\text {ts }}$ / $+; \mathrm{crq}^{-G a l 4 /}+$ and $w^{1118}$, UAS-Stat92E-IR; UAS-Stat92-IR/ + ) 5 days after injection with 500 CFU M. marinum. The value shown is the geometric mean of two ratios (knockdown/driver-only control and knockdown/STAT-IR only control). Atg2 fold change is statistically different from other autophagy genes ( ${ }^{\star \star \star} P<0.001$ by Mann-Whitney test, $n=14$ ). (d) Atg2 expression by qRT-PCR in S2R ${ }^{+}$cells overexpressing HA (control) or upd3. Normalized to Rp/1. Values are statistically different ( ${ }^{\star} P<0.05$ by Mann-Whitney test, $n=6$ ). (e) Expression of Atg2 by qRT-PCR in S2R + cells with Luciferase or Stat92E knocked down, with or without M. marinum infection. Normalized to Rpl1 and then to the uninfected Luciferase control. Values are statistically different as

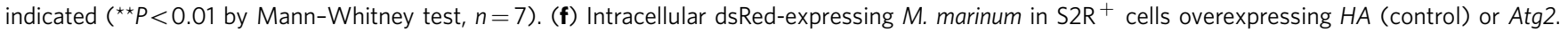
Picture is a representative; graph shows normalized M. marinum fluorescence from 37 (Atg2 O/E) or 45 (control) cells. Values are statistically different $\left({ }^{\star} P<0.05\right.$ by Mann-Whitney test). Scale bar, $5 \mu \mathrm{m}$. (g) Intracellular dsRed-expressing M. marinum in S2R ${ }^{+}$cells with Luciferase or Stat $92 \mathrm{E}$ knocked down by RNAi. The graph shows normalized M. marinum fluorescence from 44 (Stat dsRNA) or 76 (Luc dsRNA) cells. Values are statistically different $\left({ }^{\star \star} P<0.01\right.$ by Mann-Whitney test). 
distribution, both processes associated with intracellular survival or killing of mycobacteria ${ }^{33-36}$.

To clarify how cytokines interacted with other inflammatory cues to regulate phagocyte Atg2, we measured expression of Atg2 in $\mathrm{S}_{2} \mathrm{R}^{+}$cells with or without Stat92E knockdown and with or without $M$. marinum infection. Stat92E knockdown or M. marinum infection gave mild or no increase in Atg2 expression but, when combined, these two stimuli strongly
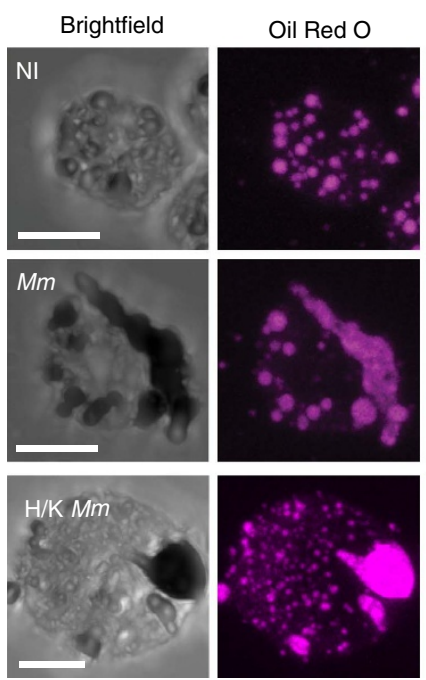

Anti-mycobacteria antibody
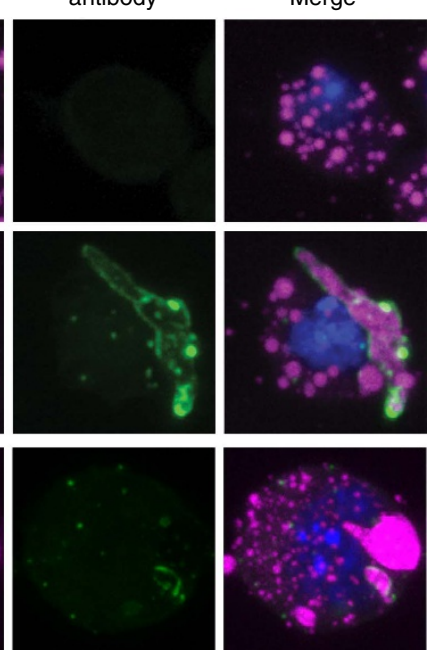

b

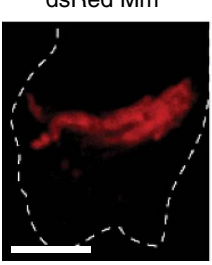

LipidTox

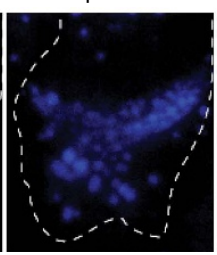

C
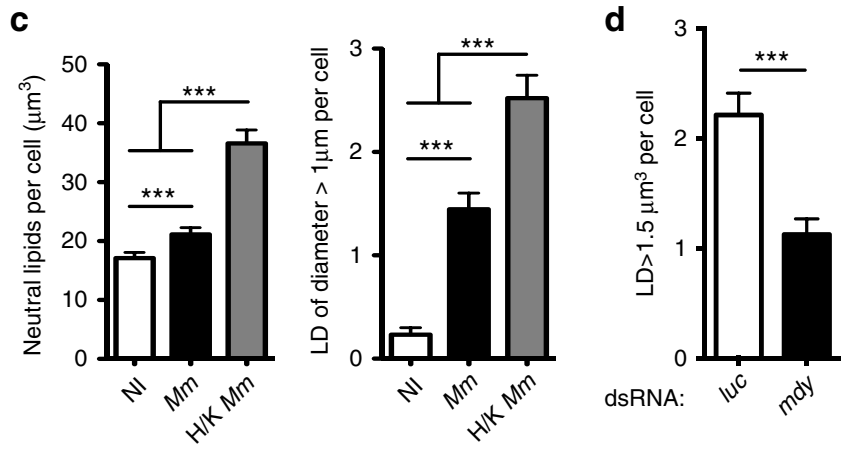

e

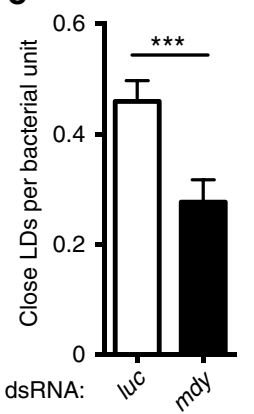

GFP-ATG2

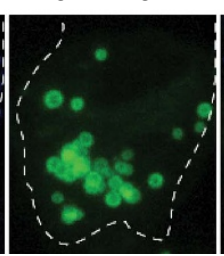

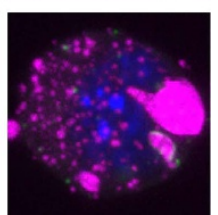

induced Atg2 expression, suggesting that upd3 limits infectioninduced Atg2 overexpression (Fig. 2e). The difference observed between the in vivo context (where infection is not required for increased Atg2 expression in Stat92E knockdowns) and these in vitro observations may be the result of the different cytokine milieu experienced by cells in culture, compared with those in the animal, or to the contribution of other tissues.

Increased Atg2 impairs intracellular M. marinum survival. As Atg2 was specifically affected by loss of upd3-Stat92E signalling, we assayed the effect of Atg2 overexpression in cultured phagocytes. Overexpression of GFP-ATG2 or RNAi of Stat92E reduced by $50 \%$ the number of intracellular mycobacteria per cell and eliminated mycobacterium-induced phagocyte death (Fig. 2f,g and Supplementary Fig. 5e,f). The effect of Atg2 on resistance to infection was not due to general changes in particle internalization, as Atg2 overexpression did not impair uptake of pHrodolabelled beads (Supplementary Fig. 5g).

Atg2 knockdown has separable effects on autophagy and lipid deposition in $\mathrm{HeLa}$ cells $^{35}$. We assayed the effect of Atg2 overexpression on autophagy and found no clear differences in autophagosome number, processing of ATG8 or association of autophagosomes with mycobacteria (Supplementary Fig. 6).

M. marinum benefits from neutral lipids in phagocytes. In M. tuberculosis infection, some macrophages accumulate LDs, which has been suggested to promote mycobacterial persistence within the host ${ }^{37,38}$. Indeed, intracellular survival of $M$. tuberculosis depends on its ability to recalibrate macrophage lipid homeostasis ${ }^{34,39}$. M. marinum infection, similar to M. tuberculosis infection, induces lipid accumulation in Drosophila cells (Fig. 3a). As we had seen little effect on general autophagy from Atg2 overexpression, we explored the interaction of Atg2 with LDs. GFP-ATG2 associated with many LDs, as previously seen in HeLa cells, and sometimes appeared to coat intracellular mycobacteria (Fig. 3b and Supplementary Fig. 6a) ( $^{35}$

Quantitative analysis of lipid deposition in infected cells revealed a mild increase in total intracellular neutral lipid, but a

Figure 3 | M. marinum driven lipid accumulation is required for intracellular growth or survival of mycobacteria. (a) Neutral lipids (stained with Oil Red O) and intracellular mycobacteria (stained with fluorescein isothiocyanate-anti-M tuberculosis) in cells variously uninfected, infected with live $M$. marinum or infected with heat-killed $(H / K) M$. marinum. (b) Co-localization of ATG2-GFP with LipidTox-stained intracellular neutral lipids. Cells were observed $48 \mathrm{~h}$ after transfection. Scale bars, $5 \mu \mathrm{m}$. (c) Quantification of neutral lipid volume per cell and total number of LDs with at least one axis $>1 \mu \mathrm{m}$ in length in cells treated as in b. Values are statistically different $\left({ }^{\star \star} P<0.01\right.$ and $\left.{ }^{\star \star \star} P<0.001\right)$ by MannWhitney test. $n=130$ for non-infected cells, $n=110$ for M.m.-infected cells and $n=96$ for cells in contact with heat-killed M.m. (d) Number of LDs (stained with BODIPY 500/510) of volume $\geq 1.5 \mu \mathrm{m}^{3}$ in cells treated with dsRNA for mdy or Luc, and infected with dsRed M. marinum. Values are statistically different ( ${ }^{\star \star \star} P<0.001$ by Mann-Whitney test, $n=71$ for $m d y$ dsRNA, $n=135$ for Luc dsRNA). (e) Number of LDs (stained with BODIPY $500 / 510$ ) at a distance $\leq 1 \mu \mathrm{m}$ from bacteria and measured per unit of bacterial volume. Cells were treated with dsRNA for mdy or Luc and infected with dsRed $M$. marinum. Values are statistically different ( ${ }^{\star \star \star} P<0.001$ by Mann-Whitney test, $n=64$ for $m d y$ dsRNA, $n=119$ for Luc dsRNA). (f) Co-localization of BODIPY-stained LDs with dsRed M. marinum in $\mathrm{S} 2 \mathrm{R}^{+}$ cells with or without $m d y$ knockdown. Scale bar, $5 \mu \mathrm{m}$. (g) Quantification of bacterial volume per cells in $\mathrm{S}_{2} \mathrm{R}^{+}$cells treated with dsRNA for mdy or Luc and infected with dsRed M. marinum. Values are statistically different ( ${ }^{\star \star \star} P<0.001$ by Mann-Whitney test, $n=64$ for mdy dsRNA, $n=119$ for Luc dsRNA). 
a

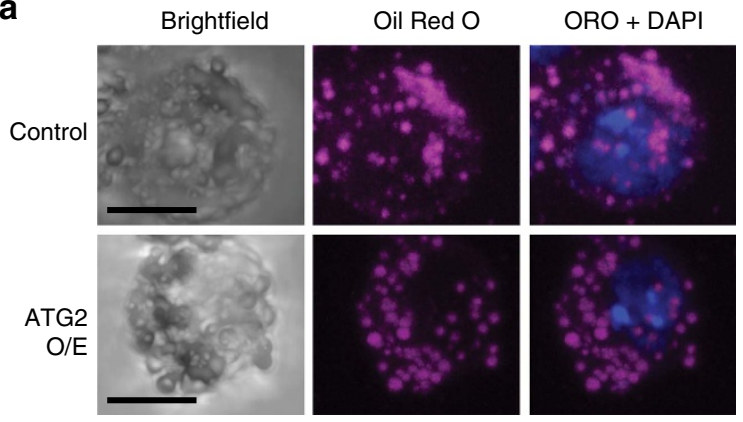

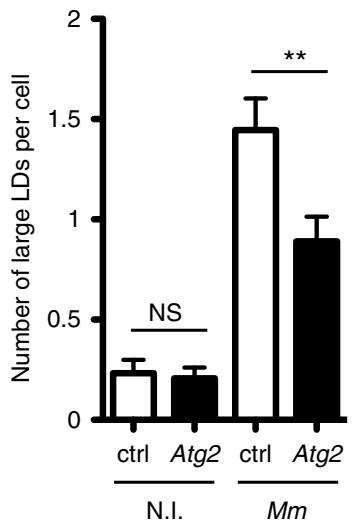

C

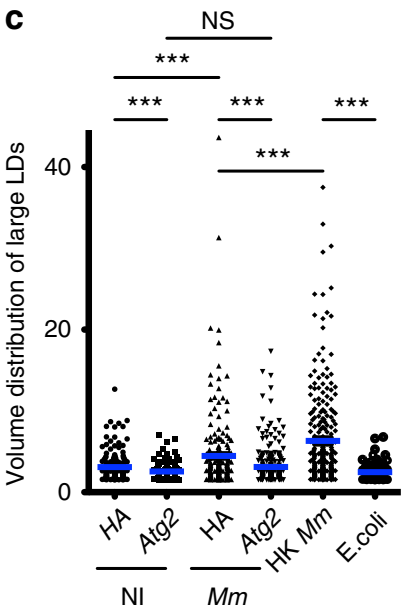

d

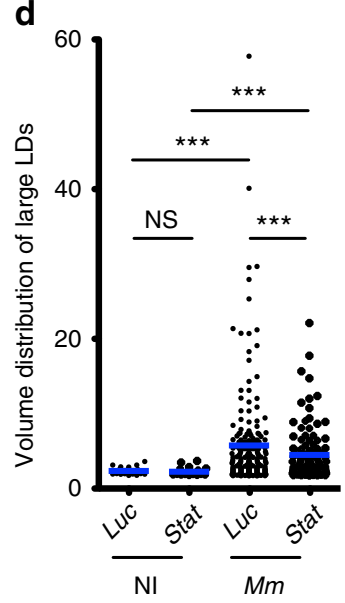

Figure 4 | Atg2 overexpression corrects LD size in M. marinum-infected cells. (a) Intracellular neutral lipid in $M$. marinum-infected cells overexpressing either HA (control) or Atg2. Scale bars, $5 \mu \mathrm{m}$.

(b) Quantification of neutral lipid volume per cell and total number of LDs with at least one axis $>1 \mu \mathrm{m}$ in length in cells treated as in $\mathbf{a}$. A slight increase in the overall volume of neutral lipids was observed in Atg2overexpressing cells compared with control $\left({ }^{\star} P<0.05\right.$ and ${ }^{\star \star} P<0.01$, Mann-Whitney test, $n=130$ for non-infected controls, $n=110$ for Mminfected controls, $n=107$ for non-infected cells overexpressing Atg 2 and $n=100$ for infected cells overexpressing Atg2). (c) Distribution of large $\left(>1.5 \mu \mathrm{m}^{3}\right) \mathrm{LD}$ volumes in cells overexpressing Atg2 or HA and with the infections indicated. Volumes $<1.5 \mu \mathrm{m}^{3}$ are not shown but were included in statistical analysis. Punctae $(3,100-5,000)$ were analysed depending on genotype and condition. Significance values are from the KolmogorovSmirnov test. (d) Distribution of large $\left(>1.5 \mu \mathrm{m}^{3}\right)$ LD volumes in cells with Luciferase or Stat92E knocked down by RNAi, with or without infection as indicated. Volumes $<1.5 \mu \mathrm{m}^{3}$ are not shown but were included in statistical analysis. Punctae (1,100 to 2,000) were analysed depending on genotype and condition. Significance values are from the KolmogorovSmirnov test.

dramatic increase in the number of unusually large LDs in infected samples (Fig. 3c). Heat-killed M. marinum induced greater accumulation of LDs and more large lipid structures than live bacteria (Fig. 3c), whereas infection of S2R ${ }^{+}$cells with Escherichia coli, as an activator of general inflammatory signals, did not alter neutral lipids (Supplementary Fig. 8a,b). Although we were able to stain neutral lipids within free mycobacteria, the size and shape of the lipid inclusions we observed in infected phagocytes were incompatible with the lipids being exclusively intrabacterial and the large irregular lipid inclusions we observed were often but not always physically associated with intracellular mycobacteria as detected by immunofluorescence or fluorescent protein expression (Fig. 3a,b and Supplementary Fig. $8 \mathrm{c}-\mathrm{e})$.

The fact that dead mycobacteria drove lipid accumulation and that large lipid inclusions were only partially associated with live intracellular $M$. marinum implied that these aberrant large lipid inclusions were host-derived and resulted from some change in host cell lipid deposition or metabolism exploited by mycobacteria. We tested this directly by RNAi inactivation of midway $(m d y)$, the Drosophila homologue of diacylglycerol $O$-acyltransferase 1 (DGAT1), the final enzyme in triglyceride synthesis ${ }^{40}$. $m d y$ knockdown reduced LD numbers and volume in uninfected and M. marinum-infected S2R + cells (Supplementary Fig. 9a-f). We identified a threshold LD volume associated with pathogenesis by determining the cumulative distribution function of LD volumes in uninfected and $M$ marinum-infected cells. These functions intersected at a volume of $1.5 \mu \mathrm{m}^{3}$, indicating that most droplets of volume greater than this in infected cells were due to mycobacterial activity (Supplementary Fig. 7g). mdy knockdown impaired $M$. marinum-induced formation of large LDs and reduced mycobacterial association with LDs, leading to a general reduction in bacterial burden per cell (Fig. 3d-g). These data demonstrate that M. marinum survival and/or proliferation is dependent on lipid accumulation in Drosophila phagocytes.

Increased Atg2 expression reduces large LDs. As preventing LD accumulation during infection reduced mycobacterial numbers and as ATG2 associated with LDs and reduced bacterial load, we hypothesized that ATG2 affected resistance to $M$. marinum by altering lipid deposition in immune cells. We tested this by infecting Atg2-transfected and control cells with M. marinum and observing LD volume (Fig. $4 \mathrm{a}-\mathrm{c}$ ). M. marinum-infected cells overexpressing Atg2 exhibited a marked reduction in the number of large LDs compared with infected controls. Stat92E knockdown, which induced Atg2 expression (Fig. 2e), also decreased large LD size in infected cells (Fig. 4d). Stat92E knockdown did not affect LD size in uninfected cells, in keeping with our observation that this knockdown did not affect Atg2 expression in the absence of infection (Fig. 2e). These data together suggest that overexpression of Atg 2 observed in upd3/Stat92E knockdown flies renders phagocytes inhospitable to mycobacteria via effects on lipid deposition.

IL-6 impairs mycobacterial killing via effects on triglyceride. To see whether our results were relevant to human mycobacterial disease, we tested the ability of several JAK-STAT activating cytokines to impair killing of Mycobacterium bovis BCG by THP1 cells. IL-6, added $2 \mathrm{~h}$ after infection, strongly inhibited the mycobactericidal activity of these cells (Fig. 5a). To further clarify the timing of this effect, we measured viable intracellular BCG in cells treated before and/or after infection with IL-6; we observed that either pre-, post- or pre- and postinfection IL- 6 caused a significant increase in viable intracellular BCG at 24 and $48 \mathrm{~h}$ 
a

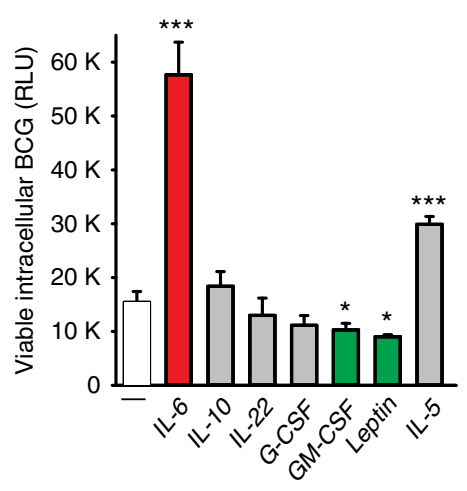

b

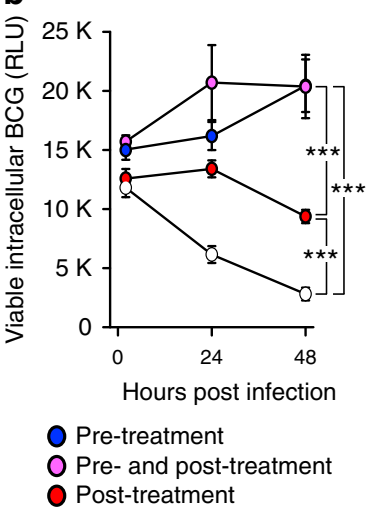

Pre- and post-treatment

O Post-treatment

d
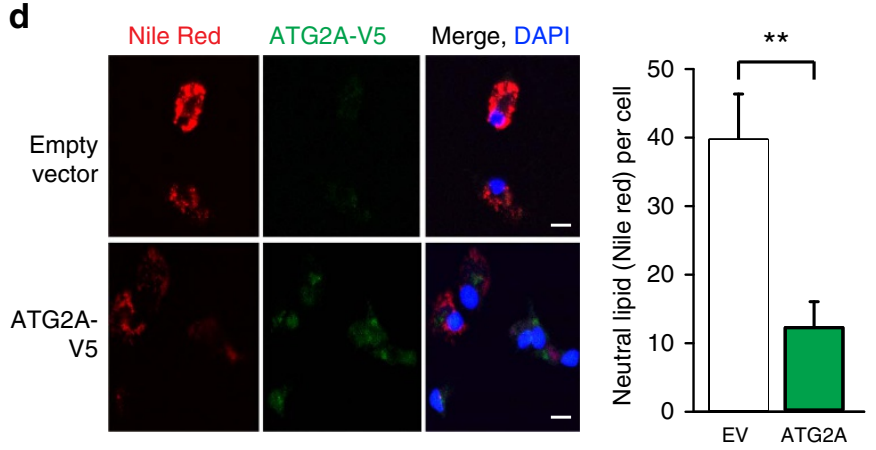

f

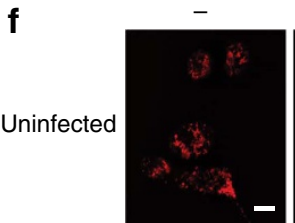

IL-6

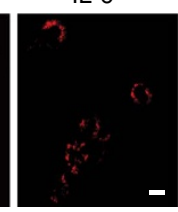

Infected $\frac{-}{4 y^{2}, x^{3}}$

IL-6

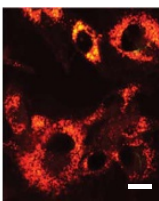

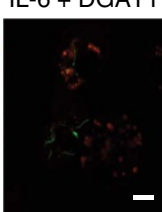

g

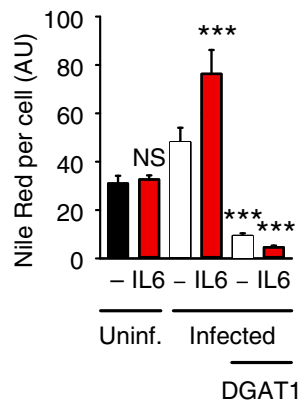

C

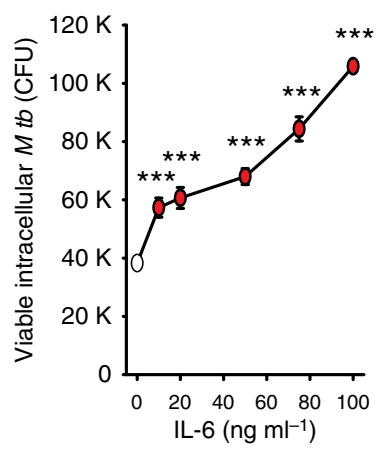

e

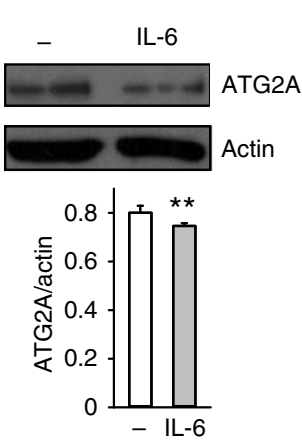

h

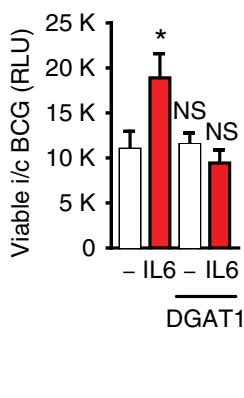

Figure 5 | IL-6 impairs intracellular killing of $\boldsymbol{M}$. tuberculosis through altered LD homeostasis. (a,b) Screening of the effect of cytokines signaling through JAK-STAT pathway on intracellular survival of mycobacteria in human macrophages. (a) Differentiated THP-1 cells were incubated with a luminescent strain of $M$. bovis BCG (BCG-lux) for $2 \mathrm{~h}$, repeatedly washed, then treated with cytokines $\left(20 \mathrm{ng} \mathrm{ml}{ }^{-1}\right)$ for $24 \mathrm{~h}$ at $37^{\circ} \mathrm{C}$. Cells were then lysed and luminescence measured. Viable intracellular mycobacteria were increased by IL-6 (red) and IL-5, and reduced by granuloyte-macrophage colonystimulating factor and Leptin (green). Significance comparisons are between indicated treatment and untreated control. (b) Comparison of effects of treatment with IL-6 before and after infection in THP-1 cells. (c) Primary human macrophages were incubated with Bleupan M. tuberculosis for $2 \mathrm{~h}$, repeatedly washed and then treated with IL-6 (red, at concentrations shown) for $24 \mathrm{~h}$ at $37^{\circ} \mathrm{C}$ before cells were lysed and plated to count CFUs. Significance comparisons are between indicated treatment and untreated control. (d) ATG2A-V5 overexpression reduces intracellular neutral lipids in THP-1 cells. Scale bars, $10 \mu \mathrm{m}$. (e) Treatment of primary human macrophages with IL-6 ( $80 \mathrm{ng} \mathrm{ml}^{-1}$ for $24 \mathrm{~h}$ ) causes a small reduction in ATG2A protein abundance. Quantification is from three independent experiments. Error bars represent s.e.m. (f,g) BCG-induced LD accumulation in enhanced by IL-6 treatment. Representative images (f) and fluorescence quantification $(\mathbf{g})$ of intracellular LDs (red), in primary human macrophages $24 \mathrm{~h}$ after infection with GFP-labelled M. bovis BCG (green). LDs are increased following infection, further increased in the presence of IL-6 treatment (80 ng ml-1; red) and significantly reduced by pretreatment with the DGAT1 inhibitor T863 $(10 \mu \mathrm{M}, 48 \mathrm{~h})$. Scale bars, $10 \mu \mathrm{m}$ (f). (h) The increase in intracellular viable $\mathrm{M}$. bovis BCG in primary human macrophages treated with IL-6 (red; $80 \mathrm{ng} \mathrm{ml}^{-1}$ for $24 \mathrm{~h}$ post infection) is blocked by pretreatment with the DGAT1 inhibitor T863 (as above). ${ }^{\star} P<0.05,{ }^{\star \star} P<0.005$ and ${ }^{\star \star \star} P<0.001$. Figures are representative of at least three independent experiments with at least three replicates per data point.

(Fig. 5b). We also observed that IL-6 caused a dose-dependent increase in survival of an auxotrophic mutant of M. tuberculosis within primary human macrophages (Fig. 5c). We then tested the effect of ATG2A overexpression on intracellular lipid stores in THP-1 cells and observed that overexpression of ATG2A was sufficient to markedly reduce neutral lipid stores in these cells (Fig. 5d). upd3 has been suggested to be an evolutionary homologue of IL-6, and IL-6 is inducible by M. tuberculosis and can prevent killing of intracellular mycobacteria $^{15,41,42}$; this suggested that the mechanisms of IL-6 action on human macrophages might be similar to the effects of upd3 in Drosophila. 


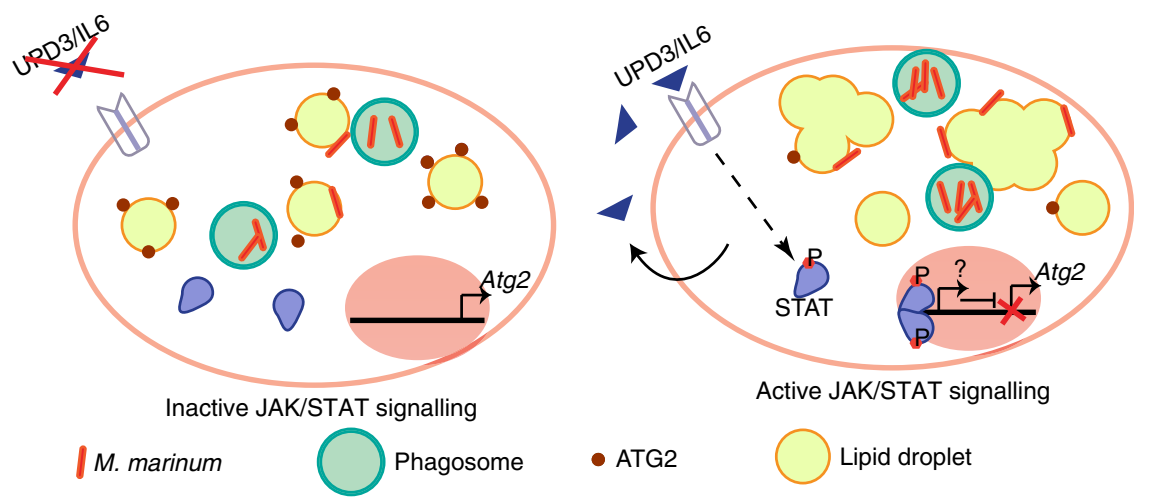

Figure 6 | A model of the role of upd3-Stat92E signalling in the M. marinum-infected phagocyte. Upon infection, haemocytes secrete upd3, which activates the JAK-STAT pathway by signalling back to haemocytes. Upon activation of JAK-STAT, Atg2 expression is reduced, large and irregular LDs are formed, and the bacteria survive and proliferate within the immune cell. However, if the JAK-STAT pathway is inhibited, Atg2 expression is increased and ATG2 protein is found surrounding LDs and bacteria (red dots). The LDs maintain small and circular structures and the bacteria are less able to survive or replicate.

We next tested the effect of IL-6 treatment on abundance of ATG2A protein. Although IL-6 treatment caused a statistically significant reduction in ATG2A protein abundance in monocytederived macrophages ( $P=0.0384$, unpaired Student's $t$-test), this effect was extremely small (Fig. 5e). Thus, although the STATATG2 transcriptional circuit may function in similar ways in human and fly macrophages, it is difficult to imagine that the effect of exogenous IL-6 on mycobacterial number is driven by reductions in total quantity of cellular ATG2A.

We then examined the effect of IL- 6 on lipid accumulation in infected primary human macrophages. We treated macrophages with IL-6 with or without pharmacological inhibition of DGAT1. IL-6 treatment potentiated the increase in intracellular lipid deposition driven by mycobacterial infection (Fig. 5f,g). DGAT1 inhibition reduced intracellular triglyceride levels, eliminated the ability of mycobacteria to drive lipid accumulation and abolished the ability of IL- 6 to promote intracellular mycobacterial survival (Fig. 5f-h), indicating that the ability of IL-6 to increase intracellular mycobacterial numbers depended on its ability to enhance bacterial access to host triglyceride.

\section{Discussion}

Here we identify a molecular mechanism that can reduce mycobacterial viability in phagocytes and show how this mechanism is inhibited by cytokine signalling in vivo. We show that haemocyte-derived cytokine signals activate JAK-STAT signalling in haemocytes in the early phases of M. marinum infection. Inhibiting production or response to this signal in adult haemocytes results in enhanced resistance to mycobacterial infection. We then show that flies in which either upd3 or JAK-STAT signalling is impaired in haemocytes exhibit increased expression of Atg2, but not other autophagy genes. Atg2 overexpression is sufficient to reduce mycobacterial survival in cultured Drosophila phagocytes and to inhibit changes in neutral lipid metabolism observed in M. marinum-infected cells. In parallel, we demonstrate that disturbing pathogen-induced changes in triglyceride metabolism directly impairs bacterial survival. Finally, a similar mechanism appears to function in human macrophages in response to IL-6 signalling. Together, these data indicate that some STAT-activating cytokines increase intracellular mycobacterial survival via effects on triglyceride deposition, that ATG2 regulation is a potential effector and that inhibition of macrophage triglyceride accumulation may be a useful therapeutic approach in mycobacterial disease.
Mycobacterium-infected macrophages often show aberrant accumulation of neutral lipids ${ }^{43}$, but the biological importance of this event is only partly understood. Intracellular mycobacteria depend on host lipids as a source of nutrients ${ }^{37,39}$, but this is not necessarily the only important role of neutral lipids in promoting mycobacterial survival. The fact that we observe changes in mycobacterial numbers as a result of triglyceride perturbations as little as $24 \mathrm{~h}$ after infection implies that accumulation of triglycerides may also directly disrupt phagocyte killing mechanisms. In this context, it may be relevant that neutral lipids have recently been observed to be protective against oxidative stress in the developing Drosophila central nervous system $^{44}$, suggesting that lipid accumulation in macrophages might protect intracellular bacteria from oxidative killing mechanisms. Nonetheless, this effect will clearly not be universal: in some contexts, LDs contain large amounts of free histones that can exert direct antibacterial properties ${ }^{45}$. Another possibility is that the effect on bacterial survival is due to changes in production of lipids used as signalling molecules, such as leukotrienes, lipoxins and other eicosanoids ${ }^{46}$. Although the roles of these molecules in Drosophila immune responses has not been characterized, they play critical roles in regulation of vertebrate responses to mycobacterial infection ${ }^{47}$ and it is not difficult to imagine that their production would be altered by the effects on triglyceride deposition we document here. In either case, our observations suggest the possibility that fusion of LDs with bacteria-containing vesicles is normally a productive aspect of the host defense that has been co-opted by some intracellular pathogens to promote their own nutrition and/or protect themselves from intracellular killing-although other explanations for these phenomena cannot be excluded.

Although autophagy can be altered by pro- and antiinflammatory cytokines and has been argued to be important in clearance of intracellular M. tuberculosis ${ }^{3,33}$, the in vivo relevance of this effect is not clear ${ }^{48}$. The role of autophagy in M. marinum infection is similarly unclear, though cytosolic $M$. marinum can be sequestered in double-membraned vesicles in bone-marrowderived macrophages independently of the classical autophagy pathway proteins ATG5 or LC3, suggesting an unusual autophagy-like mechanism may be involved in the response to this organism ${ }^{49}$. Our work suggests that the effects of autophagy on infection and sequestration of mycobacteria and the effects of mycobacteria on intracellular lipid inclusions may be fundamentally linked by common underlying molecular mechanisms. Effects ascribed to autophagic mechanisms may, 
in fact, be due to autophagy components-in the case of our work, ATG2-acting outside of the normal autophagy pathways to alter lipid accumulation. Future work, in particular to delineate the genetic requirements for lipophagy as opposed to general autophagy, will help resolve these effects in infection.

Our data suggest a model in which STAT-driven Atg2 repression deregulates lipid storage upon infection to the advantage of mycobacteria (Fig. 6). Despite potent bactericidal mechanisms, macrophages are often unable to eradicate M. tuberculosis, which then persists in the organs of the infected individual ${ }^{50}$. Therefore, novel strategies are needed to counteract bacterial latency and permit killing. Our work suggests two strategies to target mycobacterial infection by altering lipid metabolism in macrophages. One such strategy is direct inhibition of DGAT1. The fact that ATG2A overexpression is sufficient to reduce intracellular triglyceride in cultured THP-1 cells, and that Atg2 overexpression is sufficient to reduce mycobacterial survival in fly cells, suggests another such strategy. The strong and specific repression of Atg2 by JAKSTAT signalling, at least in Drosophila, indicates one regulatory mechanism for this gene, but it is clear that even in this comparatively simple system other regulators are critical. Identification of these other regulatory mechanisms may open new avenues for antimycobacterial therapies.

\section{Methods}

Drosophila stocks. The following stocks were used: from the Bloomington Drosophila Stock Center: os ${ }^{s}$ (BDSC 79), Gal80 ${ }^{t s}$ (BDSC 7017, 7018, 7019, 7108), c564 (BDSC 6982), HmlA-Gal4 (BDSC 30140) and UAS-Stat92E-IR (BDSC 26899); from the Vienna Drosophila Resource Center: UAS-upd3-IR (VDRC 106869) and UAS-Stat92E-IR (VDRC 106980); from Hervé Agaisse (Yale University): UAS-upd3-IR; from Nathalie Franc (The Scripps Research Institute): crq-Gal4; from James Castelli-Gair Hombría (Centro Andaluz de Biología del Desarrollo): UAS-dome $e^{\text {Acyt }}$.

Survival analysis. Mycobacterial and Drosophila culture and infections were carried out substantially as described, using 5- to 9-day-old male flies ${ }^{12}$. Injected flies were incubated 20 flies to a vial and the number dead was recorded twice a day; all survival experiments were repeated at least three times with qualitatively similar results. When using a tubulin-Gal80 $0^{t s}$ construct, crosses were kept at $18^{\circ} \mathrm{C}$ and flies were collected and switched to $29^{\circ} \mathrm{C}$ the day after eclosion; they were then maintained at $29^{\circ} \mathrm{C}$ for the duration of the experiment. For experiments not involving tub-Gal80 $0^{\mathrm{ts}}$, flies were kept at $25^{\circ} \mathrm{C}$ at all times.

Intravital imaging. For haemocyte counts, 5-7-day-old flies carrying $\mathrm{Hml} \mathrm{L}$-Gal4, UAS-2xeGFP or Crq-Gal4, UAS-2xeGFP were injected with 500 colony-forming unit (CFU) M. marinum and imaged at indicated times. Flies were glued to coverslips and imaged immediately using a confocal microscope (SP5, Leica) equipped with an environmental control chamber. The environmental chamber was set to $25^{\circ} \mathrm{C}$ or $29^{\circ} \mathrm{C}$ when tub-Gal $80^{\text {ts }}$ flies were used. Flies that died during the procedure were excluded from the analysis. GFP was excited at $488 \mathrm{~nm}$ with an argon laser and $5.63 \mu \mathrm{m}$ sections were imaged through the coverslip, glue and dorsal cuticle. Flies carrying Hml $\Delta$-dsRed.nls and upd3-Gal4, UAS-GFP were imaged $48 \mathrm{~h}$ after injection of 5,000 CFUs using the same procedure with sequential excitation at 488 and $561 \mathrm{~nm}$, and with images taken every $0.69 \mu \mathrm{m}$.

Drosophila cell culture and infection. $\mathrm{S}^{2} \mathrm{R}^{+}$cells (DGRC, Bloomington, Indiana) were maintained in M3 medium supplemented with $10 \%$ fetal bovine serum and $50 \mathrm{U} \mathrm{ml}^{-1}$ penicillin-streptomycin. S2R ${ }^{+}$cells were transfected using Effectene (Qiagen); $24 \mathrm{~h}$ later, they were washed and then infected with M. marinum (suspended in M3 medium supplemented with 10\% fetal bovine serum and 1\% penicillin-streptomycin) at a multiplicity of infection (MOI) of 10-20. Samples were collected after 24 or $48 \mathrm{~h}$ for western blotting, qRT-PCR or imaging.

For overexpression experiments, GFP-tagged human ATG2A (from plasmid pEGFP-C1-hAtg2A, Addgene, deposited by Noboru Mizushima) and Drosophila upd3 (by PCR from Drosophila complementary DNA) were cloned into pPAC-HA, a vector containing the Drosophila Actin 5C promoter (from Nic Tapon (Francis Crick Institute)). In all overexpression experiments, the displayed control is cells transfected in parallel with the empty vector, in which a $3 \mathrm{xHA}$ peptide is expressed.

Phagocytosis assays on cultured cells were done as described ${ }^{51}$

For RNAi experiments, cells were bathed in double-stranded RNA (dsRNA) targeting Stat92E or, as a control, Renilla luciferase. RNAi constructs were designed using the SnapDragon web tool with no predicted off-targets; template amplicons were produced by PCR from plasmid or Drosophila genomic DNA with the following primers: T7-RLucL, 5' $5^{\prime}$ TAA TAC GAC TCA CTA TAG GGA GAC TGA TCA AGA GCG AAG AGG G-3'; T7-RLucR, $5^{\prime}$-TAA TAC GAC TCA CTA TAG GGA GAC TTT CAC GAA CTC GGT GTT G-3'; T7-Stat92eL, $5^{\prime}$-TAA TAC GAC TCA CTA TAG GGA GAA AGC TGC TTG CCC AAA ACT A-3'; T7-Stat92eR, 5'-TAA TAC GAC TCA CTA TAG GGA GAG TCG ACG ATA AAG GCA GAG C-3'; T7-mdyL, 5' -TAA TAC GAC TCA CTA TAG GGA GAG CGA CTT CTT AAA CTT GCG G-3'; and T7-mdyR, 5' -TAA TAC GAC TCA CTA TAG GGA GAG ATA TGG CCG ATA GGG GAA T-3'. dsRNA was made with the MEGAscript T7 kit (Ambion). For efficient knockdown, $1.2 \mu \mathrm{g}$ dsRNA was added per well in 48-well plates containing 200,000-500,000 cells according to the DRSC RNAi protocol. After 3 days of dsRNA treatment, $M$. marinum was added to the dsRNA solution, at an MOI of 20-60 based on the initial number of cells added in the experiment. Cells were lysed in TRIzol $24-48 \mathrm{~h}$ post infection.

Flow cytometry of Drosophila haemocytes and S2R + cells. For cytotoxicity assays in uninfected and infected Drosophila S2R + cells, the supernatant medium containing non-adherent cells was collected $24 \mathrm{~h}$ post infection and transferred directly into a FACS tube. The adherent S2R + cells of the same sample were detached using $2 \mathrm{ml}$ of ice-cold culture medium and pipetting up and down. The medium was then added to the previously collected supernatant sample. All samples were filtered with a $70 \mu \mathrm{m}$ cell strainer filter (BD Biosciences) and kept on ice before FACS analysis. Five minutes before analysis, $5 \mu \mathrm{l}$ of propidium iodide (PI) was added to $500 \mu \mathrm{l}$ of samples to stain dead cells. Cells were analysed with a FACS Aria II (Becton Dickinson (BD)), using a $100 \mu \mathrm{m}$ nozzle and the FACS-Diva software. All experiments included the following controls: $M$. marinum only samples with and without PI staining, and non-infected cells with and without PI. For phagocytosis assays with phrodo-coated beads in uninfected and infected Drosophila S2R + cells, supernatant and adherent cells were collected as described above $48 \mathrm{~h}$ post transfection. Cells $(500,000)$ were spun down for $3 \mathrm{~min}$ at $21^{\circ} \mathrm{C}$, 1,400 r.p.m. and resuspended in fresh medium. pHrodo-coated beads were added at a concentration of $1 / 100 \mathrm{v} / \mathrm{v}$ and incubated with cells for $30 \mathrm{~min}$ at $25^{\circ} \mathrm{C}$. Cells were then spun for $3 \mathrm{~min}$ at $21^{\circ} \mathrm{C}, 1,400$ r.p.m. to remove free beads. Fresh medium was added and cells were incubated one more hour before analysis by FACS (BD LRS Fortessa). Samples were analysed in the ultraviolet channel (IndoViolet beads) and the Texas red channel (pHrodo). The experiment included the following controls: cells without beads and beads-only control (no pHrodo).

For measurement of M. marinum uptake by haemocytes in vivo, the different genotypes $(\mathrm{Hml} \Delta \mathrm{eGFP} /+$ and $\mathrm{Hml} \Delta \mathrm{eGFP}>\mathrm{upd} 3-\mathrm{IR})$ were injected with M. marinum expressing Tomato fluorescent protein. Sixteen hours post infection 30-40 flies per sample were anaesthetized with $\mathrm{CO}_{2}$. The flies were carefully transferred onto a $70 \mu \mathrm{m}$ mesh, and smashed and filtered in $10 \mathrm{ml}$ ice-cold $1 \times \mathrm{PBS}$ with $2 \mathrm{mM}$ EDTA. The cell suspension was centrifuged at $150 \mathrm{~g}$ for $10 \mathrm{~min}$ at $4^{\circ} \mathrm{C}$ and the supernatant carefully removed. The cell pellet was washed in $10 \mathrm{ml} 1 \mathrm{xPBS}$ with $2 \mathrm{mM}$ EDTA twice, afterwards the cells were resuspended in $500 \mu \mathrm{l}$ of $1 \times$ PBS with $2 \mathrm{mM}$ EDTA and transferred to a FACS tube by filtering the solution through a $70 \mu \mathrm{m}$ filter mesh. Samples were acquired with a BD Fortessa and analysed using the FlowJo analysis software. Four samples per genotype were analysed.

In vitro mycobacterial infection assays. Luminescent reporter strain infection: a validated luminescent reporter strain of $M$. bovis BCG (BCG-lux) encoding the Vibrio lux $A B$ gene, generated as described ${ }^{52}$, was used to infect macrophages. Correlation between CFUs and luminescence was confirmed before experiments. Primary monocyte-derived human macrophages, generated (as previously described $^{53}$ ) from healthy consented subjects (Regional Ethics approval: REC: $12 / \mathrm{WA} / 0148$ and 4/EE/1187) or THP-1 cells (ATCC), were differentiated by treatment with $5 \mathrm{ng} \mathrm{ml}^{-1}$ PMA $48 \mathrm{~h}$ before infection, inoculated with BCG-lux (at an MOI of 5:1) for $2 \mathrm{~h}$ at $37^{\circ} \mathrm{C}$, repeatedly washed, then incubated for $24 \mathrm{~h}$ at $37^{\circ} \mathrm{C}$ in the presence of compounds as indicated. Unless otherwise stated, recombinant cytokines (Peprotech) were used at $20 \mathrm{ng} \mathrm{ml}^{-1}$ and added to cells following $2 \mathrm{~h}$ incubation with mycobacteria. Where indicated, cells were pretreated with DGAT1 inhibitor T863 $(10 \mu \mathrm{M})$ for $48 \mathrm{~h}$ and then $24 \mathrm{~h}$ post infection, IL-6 was treated for $24 \mathrm{~h}$ post infection at $80 \mathrm{ng}^{-1} \mathrm{l}^{-1}$. Cells were lysed and luminescence measured as described ${ }^{53}$. Experiments were carried out in sextuplicate. Results are representative of at least three separate experiments.

M. tuberculosis infection: M. tuberculosis $\Delta$ leuD $\Delta$ panCD (Bleupan) ${ }^{54}$ stably transfected with pMSP12::GFP was grown in Middlebrook 7H9 broth (Difco) containing $0.5 \%$ glycerol, $0.05 \%$ Tween $80,10 \%$ OADC (BD), $0.05 \mathrm{mg} \mathrm{ml}^{-1}$ L-leucine, $0.024 \mathrm{mg} \mathrm{ml}^{-1}$ calcium pantothenate and $0.2 \mathrm{mg} \mathrm{ml}^{-1}$ Hygromycin B in $250 \mathrm{ml}$ sterile disposable Erlenmeyer flasks with mild agitation at $37^{\circ} \mathrm{C}$. Bacterial cells were harvested at mid $\log$ phase by centrifugation at $3,000 \mathrm{~g}$ for $7 \mathrm{~min}$, pellets were resuspended in $7 \mathrm{H} 9$ broth containing leucine and pantothenate supplements as above plus $18 \%$ glycerol and frozen at $-80^{\circ} \mathrm{C}$ until use. Cells were infected at an MOI of 5:1, lysed at times indicated and CFUs enumerated as described ${ }^{55}$.

Confocal immunofluorescence on human cells. Primary human macrophages were grown on glass coverslips, infected with M. bovis BCG (BCG-lux) and treated as described, rinsed with PBS, fixed with methanol and permeabilized with $0.1 \%$ Triton X-100 (Sigma) ${ }^{56}$ before being stained with Nile red 1:10,000 (dissolved in 
isopropanol), rinsed with water and then mounted with ProLong Gold antifade containing DAPI (Invitrogen). Images were acquired on a Zeiss LSM880 confocal microscope (Plan-Apochromat $\times 63 / 1.40$ Oil-immersion lens) and analysed with Zen 2010 (Carl Zeiss), and fluorescence per cell measured by Volocity software.

Antibodies and western blots. For the anti-ATG2A blotting shown in Fig. 5, primary human macrophages were treated with IL- $680 \mathrm{ng} \mu \mathrm{l}^{-1}$ for $24 \mathrm{~h}$ before being lysed. The primary antibody used was anti-ATG2A pAb (MBL Life Science) at 1:400. Other western blots were performed with anti-Drosophila Atg8 (courtesy Katja Köhler (University of Zürich) ${ }^{57}$ and G. Juhasz (Eötvös Loránd University) ${ }^{58}$, both used at 1:200) and anti- $\alpha$-tubulin (Developmental Studies Hybridoma Bank $12 \mathrm{G} 10$, used 1:10,000). Immunofluorescence on fly cells was performed with fluorescein isothiocyanate-labelled anti- $M$ tuberculosis (Invitrogen PA1-28997, used 1:50) and rabbit anti-GFP (Invitrogen A-11122, used 1:100). Immunofluorescence on human cells was performed with anti-V5 from Novus Biologicals (NB 600-381), 1:400.

M. marinum culture. M. marinum strain $\mathrm{M}$ and fluorescent derivatives were grown standing at $25^{\circ}$ in Middlebrook $7 \mathrm{H} 9$ media, supplemented with OADC, Tween-80, glycerol and antibiotics as necessary to maintain fluorescent plasmids ${ }^{12}$. Single-cell bacterial suspensions were produced as follows. Cultures were pelleted by centrifugation at $4,000 \mathrm{~g}$ for $5 \mathrm{~min}$, then resuspended in PBS $+0.2 \%$ Tween- 80 . Clumps were then separated by centrifugation at $200 \mathrm{~g}$ for $5 \mathrm{~min}$. The supernatant from this centrifugation contained primarily single bacterial cells. M. marinum strains were all strain $M$ carrying various fluorescent proteins under the control of the $m s p 12$ promoter $^{59}$; all were courtesy of Lalita Ramakrishnan and Antonio Pagan (University of Cambridge).

Statistics. Statistical analyses were performed using GraphPad Prism or R. Twoway analysis of variance, Mann-Whitney test and Kolmogorov-Smirnov test were used in this study. Statistical analysis was only performed on experiments with a least four samples per condition. $P$-values are as follows: ${ }^{\star} P<0.05,{ }^{*} P<0.01$ and ${ }^{* * *} P<0.001$. Unless otherwise noted, error bars correspond to s.e.m.

qRT-PCR analysis. RNA was extracted from adult flies using TRIzol (Invitrogen) according to the manufacturer's directions, except that samples from which mycobacterial RNA was amplified were first homogenized in a 3:1 mixture of chloroform and methanol ${ }^{60}$. Mycobacterial quantification was performed using a standard in which known quantities of fly and bacteria were mixed and then treated the same as injected samples. qRT-PCR was performed as described ${ }^{10,61}$ PCR primers used in this work are listed in Supplementary Table 1.

Data availability. All relevant data are available from the authors, except where precluded by human subject confidentiality.

\section{References}

1. O'Garra, A. et al. The immune response in tuberculosis. Annu. Rev. Immunol. 31, 475-527 (2013).

2. Cooper, A. M., Mayer-Barber, K. D. \& Sher, A. Role of innate cytokines in mycobacterial infection. Mucosal Immunol. 4, 252-260 (2011).

3. Harris, J. et al. T helper 2 cytokines inhibit autophagic control of intracellular Mycobacterium tuberculosis. Immunity 27, 505-517 (2007).

4. O'Shea, J. J. \& Plenge, R. JAK and STAT signaling molecules in immunoregulation and immune-mediated disease. Immunity 36, 542-550 (2012).

5. Redford, P. S., Murray, P. J. \& O'Garra, A. The role of IL-10 in immune regulation during $M$. tuberculosis infection. Mucosal Immunol. 4, 261-270 (2011).

6. Wallis, R. S. \& Hafner, R. Advancing host-directed therapy for tuberculosis. Nat. Rev. Immunol. 15, 255-263 (2015).

7. MacMicking, J. D. Cell-autonomous effector mechanisms against Mycobacterium tuberculosis. Cold Spring Harb. Perspect. Med. 4, a018507.

8. Lemaitre, B. \& Hoffmann, J. The host defense of Drosophila melanogaster. Annu. Rev. Immunol. 25, 697-743 (2007).

9. Evans, C. J., Hartenstein, V. \& Banerjee, U. Thicker than blood: conserved mechanisms in Drosophila and vertebrate hematopoiesis. Dev. Cell 5, 673-690 (2003).

10. Clark, R. I., Woodcock, K. J., Geissmann, F., Trouillet, C. \& Dionne, M. S. Multiple TGF- $\beta$ superfamily signals modulate the adult Drosophila immune response. Curr. Biol. 21, 1672-1677 (2011).

11. Ganesan, S., Aggarwal, K., Paquette, N. \& Silverman, N. NF-kappaB/Rel proteins and the humoral immune responses of Drosophila melanogaster. Curr. Top. Microbiol. Immunol. 349, 25-60 (2011).

12. Dionne, M. S., Ghori, N. \& Schneider, D. S. Drosophila melanogaster is a genetically tractable model host for Mycobacterium marinum. Infect. Immun. 71, 3540-3550 (2003).
13. Tobin, D. M. \& Ramakrishnan, L. Comparative pathogenesis of Mycobacterium marinum and Mycobacterium tuberculosis. Cell. Microbiol. 10, 1027-1039 (2008).

14. Agaisse, H., Petersen, U. M., Boutros, M., Mathey-Prevot, B. \& Perrimon, N. Signaling role of hemocytes in Drosophila JAK/STAT-dependent response to septic injury. Dev. Cell 5, 441-450 (2003).

15. Oldefest, M. et al. Upd3--an ancestor of the four-helix bundle cytokines. Biochem. Biophys. Res. Commun. 436, 66-72 (2013).

16. Brown, S., Hu, N. \& Hombria, J. C. Identification of the first invertebrate interleukin JAK/STAT receptor, the Drosophila gene domeless. Curr. Biol. 11, 1700-1705 (2001).

17. Dostert, C. et al. The Jak-STAT signaling pathway is required but not sufficient for the antiviral response of Drosophila. Nat. Immunol. 6, 946-953 (2005).

18. Krzemien, J. et al. Control of blood cell homeostasis in Drosophila larvae by the posterior signalling centre. Nature 446, 325-328 (2007).

19. Buchon, N., Broderick, N. A., Chakrabarti, S. \& Lemaitre, B. Invasive and indigenous microbiota impact intestinal stem cell activity through multiple pathways in Drosophila. Genes Dev. 23, 2333-2344 (2009).

20. Buchon, N., Broderick, N. A., Poidevin, M., Pradervand, S. \& Lemaitre, B. Drosophila intestinal response to bacterial infection: activation of host defense and stem cell proliferation. Cell Host Microbe 5, 200-211 (2009).

21. Jiang, H. et al. Cytokine/Jak/Stat signaling mediates regeneration and homeostasis in the Drosophila midgut. Cell 137, 1343-1355 (2009).

22. Osman, D. et al. Autocrine and paracrine unpaired signaling regulate intestinal stem cell maintenance and division. J. Cell Sci. 125, 5944-5949 (2012).

23. Zhou, F., Rasmussen, A., Lee, S. \& Agaisse, H. The UPD3 cytokine couples environmental challenge and intestinal stem cell division through modulation of JAK/STAT signaling in the stem cell microenvironment. Dev. Biol. 373, 383-393 (2013).

24. Wang, L. et al. Pleiotropy of the Drosophila JAK pathway cytokine Unpaired 3 in development and aging. Dev. Biol. 395, 218-231 (2014).

25. Olofsson, B. \& Page, D. T. Condensation of the central nervous system in embryonic Drosophila is inhibited by blocking hemocyte migration or neural activity. Dev. Biol. 279, 233-243 (2005).

26. Agaisse, H. \& Perrimon, N. The roles of JAK/STAT signaling in Drosophila immune responses. Immunol. Rev. 198, 72-82 (2004).

27. Makki, R. et al. A short receptor downregulates JAK/STAT signalling to control the Drosophila cellular immune response. PLoS Biol. 8, e1000441 (2010).

28. McGuire, S. E., Mao, Z. \& Davis, R. L. Spatiotemporal gene expression targeting with the TARGET and gene-switch systems in Drosophila. Sci. STKE 2004, pl6 (2004).

29. Simeone, R. et al. Phagosomal rupture by Mycobacterium tuberculosis results in toxicity and host cell death. PLoS Pathog. 8, e1002507 (2012).

30. Behar, S. M. et al. Apoptosis is an innate defense function of macrophages against Mycobacterium tuberculosis. Mucosal Immunol. 4, 279-287 (2011).

31. Chen, M., Gan, H. \& Remold, H. G. A mechanism of virulence: virulent Mycobacterium tuberculosis strain H37Rv, but not attenuated H37Ra, causes significant mitochondrial inner membrane disruption in macrophages leading to necrosis. J. Immunol. 176, 3707-3716 (2006).

32. Bina, S., Wright, V. M., Fisher, K. H., Milo, M. \& Zeidler, M. P. Transcriptional targets of Drosophila JAK/STAT pathway signalling as effectors of haematopoietic tumour formation. EMBO Rep. 11, 201-207 (2010)

33. Gutierrez, M. G. et al. Autophagy is a defense mechanism inhibiting BCG and Mycobacterium tuberculosis survival in infected macrophages. Cell 119, 753-766 (2004)

34. Singh, V. et al. Mycobacterium tuberculosis-driven targeted recalibration of macrophage lipid homeostasis promotes the foamy phenotype. Cell Host Microbe 12, 669-681 (2012).

35. Velikkakath, A. K., Nishimura, T., Oita, E., Ishihara, N. \& Mizushima, N. Mammalian Atg2 proteins are essential for autophagosome formation and important for regulation of size and distribution of lipid droplets. Mol. Biol. Cell 23, 896-909 (2012).

36. Manzanillo, P. S. et al. The ubiquitin ligase parkin mediates resistance to intracellular pathogens. Nature 501, 512-516 (2013).

37. Peyron, P. et al. Foamy macrophages from tuberculous patients' granulomas constitute a nutrient-rich reservoir for M. tuberculosis persistence. PLoS Pathog. 4, e1000204 (2008).

38. Russell, D. G., Cardona, P. J., Kim, M. J., Allain, S. \& Altare, F. Foamy macrophages and the progression of the human tuberculosis granuloma. Nat. Immunol. 10, 943-948 (2009).

39. Daniel, J., Maamar, H., Deb, C., Sirakova, T. D. \& Kolattukudy, P. E. Mycobacterium tuberculosis uses host triacylglycerol to accumulate lipid droplets and acquires a dormancy-like phenotype in lipid-loaded macrophages. PLoS Pathog. 7, e1002093 (2011).

40. Buszczak, M., Lu, X., Segraves, W. A., Chang, T. Y. \& Cooley, L. Mutations in the midway gene disrupt a Drosophila acyl coenzyme A: diacylglycerol acyltransferase. Genetics 160, 1511-1518 (2002). 
41. Nagabhushanam, V. et al. Innate inhibition of adaptive immunity: mycobacterium tuberculosis-induced IL-6 inhibits macrophage responses to IFN-gamma. J. Immunol. 171, 4750-4757 (2003).

42. Martinez, A. N., Mehra, S. \& Kaushal, D. Role of interleukin 6 in innate immunity to Mycobacterium tuberculosis infection. J. Infect. Dis. 207, 1253-1261 (2013).

43. Garton, N. J., Christensen, H., Minnikin, D. E., Adegbola, R. A. \& Barer, M. R. Intracellular lipophilic inclusions of mycobacteria in vitro and in sputum. Microbiology 148, 2951-2958 (2002).

44. Bailey, A. P. et al. Antioxidant role for lipid droplets in a stem cell niche of Drosophila. Cell 163, 340-353 (2015).

45. Anand, P. et al. A novel role for lipid droplets in the organismal antibacterial response. elife 1, e00003 (2012).

46. Tobin, D. M. \& Ramakrishnan, L. TB: the Yin and Yang of lipid mediators. Curr. Opin. Pharmacol. 13, 641-645 (2013).

47. Tobin, D. M. et al. The lta4h locus modulates susceptibility to mycobacterial infection in zebrafish and humans. Cell 140, 717-730 (2010).

48. Kimmey, J. M. et al. Unique role for ATG5 in neutrophil-mediated immunopathology during M. tuberculosis infection. Nature 528, 565-569 (2015).

49. Collins, C. A. et al. Atg5-independent sequestration of ubiquitinated mycobacteria. PLoS Pathog. 5, e1000430 (2009).

50. Flynn, J. L. \& Chan, J. Tuberculosis: latency and reactivation. Infect. Immun. 69, 4195-4201 (2001).

51. Colas, C. et al. An improved flow cytometry assay to monitor phagosome acidification. J. Immunol. Methods 412, 1-13 (2014).

52. Kampmann, B. et al. Evaluation of human antimycobacterial immunity using recombinant reporter mycobacteria. J. Infect. Dis. 182, 895-901 (2000)

53. Renna, M. et al. Azithromycin blocks autophagy and may predispose cystic fibrosis patients to mycobacterial infection. J. Clin. Invest. 121, 3554-3563 (2011).

54. Sampson, S. L. et al. Protection elicited by a double leucine and pantothenate auxotroph of Mycobacterium tuberculosis in guinea pigs. Infect. Immun. 72, 3031-3037 (2004).

55. Schiebler, M. et al. Functional drug screening reveals anticonvulsants as enhancers of mTOR-independent autophagic killing of Mycobacterium tuberculosis through inositol depletion. EMBO Mol. Med. 7, 127-139 (2015).

56. Floto, R. A. et al. Dendritic cell stimulation by mycobacterial Hsp70 is mediated through CCR5. Science 314, 454-458 (2006).

57. Barth, J. M., Szabad, J., Hafen, E. \& Kohler, K. Autophagy in Drosophila ovaries is induced by starvation and is required for oogenesis. Cell Death Differ. 18, 915-924 (2011).

58. Nagy, P. et al. Atg17/FIP200 localizes to perilysosomal Ref(2)P aggregates and promotes autophagy by activation of Atg1 in Drosophila. Autophagy 10, 453-467 (2014).

59. Chan, K. et al. Complex pattern of Mycobacterium marinum gene expression during long-term granulomatous infection. Proc. Natl Acad. Sci. USA 99, 3920-3925 (2002)

60. Alland, D. et al. Identification of differentially expressed mRNA in prokaryotic organisms by customized amplification libraries (DECAL): the effect of isoniazid on gene expression in Mycobacterium tuberculosis. Proc. Natl Acad. Sci. USA 95, 13227-13232 (1998).
61. Dionne, M. S., Pham, L. N., Shirasu-Hiza, M. \& Schneider, D. S. Akt and FOXO dysregulation contribute to infection-induced wasting in Drosophila. Curr. Biol. 16, 1977-1985 (2006).

\section{Acknowledgements}

We thank Hervé gAaisse, James Castelli-Gair Hombría, Martin Zeidler, Bruno Lemaitre Bruce Edgar, Teresa Żoładek, Lalita Ramakrishnan, the Vienna Drosophila RNAi Center and the Bloomington Drosophila Stock Center for flies or plasmids; Gabor Juhasz, Katja Köhler and Sara Cherry for antibodies to Drosophila ATG8; Brian Robertson, Bill Jacobs and Lalita Ramakrishnan for bacterial strains; Karen Liu, Serge Mostowy, Frédéric Geissmann, Chloë Colas, Katie Woodcock and members of the Dionne and Stramer laboratories for helpful discussion and comments. C.B.P. was supported by a $\mathrm{PhD}$ studentship from the Oliver Bird Rheumatism Programme. Early stages of this work were supported by grants from the BBSRC (BB/E02128X/1) and the Wellcome Trust (WT085119MA). Current work in the Dionne lab is funded by the BBSRC (BB/L020122/1) and MRC (MR/L018802/1)

\section{Author contributions}

Study conception and design: M.S.D. and C.B.P. Acquisition of data: C.B.P., M.S., S.W.S.T., J.A.S., K.K., K.P.B., M.C.M., S.M., M.P., K.P.B. and M.S.D. Analysis and interpretation of data: C.B.P., M.S., P.G., B.M.S., R.A.F. and M.S.D. Drafting of manuscript: C.B.P. and M.S.D. Critical revision: M.S.D., R.A.F. and C.B.P.

\section{Additional information}

Supplementary Information accompanies this paper at http://www.nature.com/ naturecommunications

Competing financial interests: The authors declare no competing financial interests.

Reprints and permission information is available online at http://npg.nature.com/ reprintsandpermissions/

How to cite this article: Péan, C. B. et al. Regulation of phagocyte triglyceride by a STAT-ATG2 pathway controls mycobacterial infection. Nat. Commun. 8, 14642 doi: 10.1038/ncomms14642 (2017).

Publisher's note: Springer Nature remains neutral with regard to jurisdictional claims in published maps and institutional affiliations.

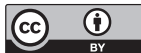

This work is licensed under a Creative Commons Attribution 4.0 International License. The images or other third party material in this article are included in the article's Creative Commons license, unless indicated otherwise in the credit line; if the material is not included under the Creative Commons license, users will need to obtain permission from the license holder to reproduce the material To view a copy of this license, visit http://creativecommons.org/licenses/by/4.0/

(C) The Author(s) 2017 